\title{
Interactive Simulation of Flexible Needle Insertions Based on Constraint Models
}

\author{
Christian Duriez ${ }^{1}$, Christophe Guébert ${ }^{1}$, Maud Marchal ${ }^{2}$, Stephane Cotin ${ }^{1}$, \\ and Laurent Grisoni ${ }^{1}$ \\ 1 INRIA Nord-Europe (University of Lille), France \\ 2 INRIA Bretagne Nord-Atlantique (IRISA-INSA), France \\ christian.duriez@inria.fr
}

\begin{abstract}
This paper presents a new modeling method for the insertion of needles and more generally thin and flexible medical devices into soft tissues. Several medical procedures rely on the insertion of slender medical devices such as biopsy, brachytherapy, deep-brain stimulation. In this paper, the interactions between soft tissues and flexible instruments are reproduced using a set of dedicated complementarity constraints. Each constraint is positionned and applied to the deformable models without requiring any remeshing. Our method allows for the 3D simulation of different physical phenomena such as puncture, cutting, static and dynamic friction at interactive frame rate. To obtain realistic simulation, the model can be parametrized using experimental data. Our method is validated through a series of typical simulation examples and new more complex scenarios.
\end{abstract}

\section{Introduction}

Needles, electrode or biopsy tools are some examples of thin and flexible medical tools used in a clinical routine. Several medical applications are concerned by the use of these tools, such as biopsy, brachytherapy or deep brain stimulation. As these objects are often thin and flexible, the accuracy of their insertion into soft tissues can be affected. Moreover, different physical phenomena, such as puncture, friction or cutting through heterogeneous tissues could alter the procedure. The simulation of the insertion of thin and flexible medical tools into various tissues can enable useful clinical feedback, such as training but also planning. We chose the example of a needle to explain our methodology but our method can be generalized to thin and flexible instruments.

Pioneering works concerning needle insertion were presented by Di Maio et al. 1] and Alterovitz et al. 2]. They proposed modeling methods based on FEM for the interaction between a needle and soft tissues. A recent survey proposed by Abolhassani et al. 3 summarizes the different characteristics of the existing methods in the literature. Remeshing process of tissue models remains an obstacle to obtain interactive simulations. In 4, the authors simulate the insertion of several rigid needles in a single soft tissue (such as during brachytherapy procedures). The interaction model between needle and tissue is the most challenging 
part as it combines different physical phenomena. Different studies based on experimental data are proposed in the literature to identify the forces occuring during the needle insertion [5]. Three different types of force are often underlined: puncture force, cutting force and friction force. Recent studies use experimental data to perform an identification of the model parameters $6,7,8$.

In this paper, a new generic method based on the formulation of several constraints is proposed in order to simulate the insertion of thin and flexible medical devices into soft tissues. Any classical deformation model of both tissue and needle can be used with our approach. For this study, we obtain interactive frame rate while modeling the geometrical non-linearities of the tissue and needle deformations. Contrary to existing methods, no remeshing process is needed, even if Finite Element Method (FEM) is used for the tissue simulation. Our method can handle complex scenarios where needle steering, non-homogeneous tissues and interactions between different needles can be combined.

The paper is divided into five sections. In Section 2] the formulation of our modeling method is presented. Section 3 details the simulation process. Section 4 shows the results obtained with the new modeling method. Section 5 draws conclusions and discusses future works.

\section{Constraint-Based Modeling of Needle Insertion}

In this work, we propose a new model for the interactions that take place at the surface of a needle during its insertion in soft tissues. The formulation relies on a constraint formulation which is independent of both needle and tissue models that are used to simulate deformations. We highlight two different aspects: Firstly, for the constraint positioning, no remeshing is necessary. Secondly, we present several new constraint laws, based on complementarity theory [9]. These laws capture in a unified formalism all the non-smooth mechanical phenomena that occur during insertion.

\subsection{How to Avoid Remeshing?}

The constraint positioning is defined by two points: one on the tissue volume $P$ and one on the needle curve $Q$. For each constraint, $\delta$ measures the distance between these two points along a defined constraint direction $\mathbf{n}$. The points can be placed anywhere in the volume of the soft tissue and anywhere on the needle curve. The displacements of these points are mapped with the same interpolation than the deformable models (see Fig. 1). The variation of $\delta$ can be mapped on the displacements of deformable model nodes $\Delta \mathbf{q}_{n}$ (needle) and $\Delta \mathbf{q}_{t}$ (tissue):

$$
\Delta \delta=\mathbf{n}^{T}\left(\mathbf{u}_{n}-\mathbf{u}_{t}\right)=\mathbf{n}^{T}\left(\mathbf{J}_{n} \Delta \mathbf{q}_{n}-\mathbf{J}_{t} \Delta \mathbf{q}_{t}\right)=\mathbf{H}_{n} \Delta \mathbf{q}_{n}+\mathbf{H}_{t} \Delta \mathbf{q}_{t}
$$

In the following, $\lambda$ represents the force used to solve the constraint (applied by the tissue on the needle). To map this force on the deformable model nodes, we use the virtual work principle $\left(\lambda^{T} \Delta \delta=\mathbf{f}_{t}^{T} \Delta \mathbf{q}_{t}+\mathbf{f}_{n}^{T} \Delta \mathbf{q}_{n}\right)$. We obtain:

$$
\mathbf{f}_{t}=\mathbf{H}_{t}^{T} \lambda \quad \text { and } \quad \mathbf{f}_{n}=\mathbf{H}_{n}^{T} \lambda
$$



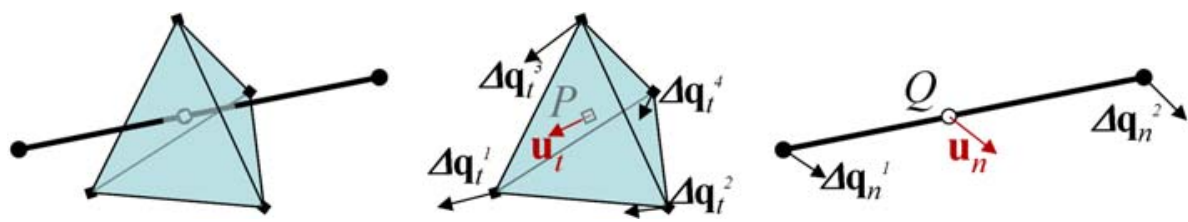

Fig. 1. Example of mapping for a constraint point. A linear interpolation is used on tetrahedra for the soft tissue model, the displacement $\mathbf{u}_{t}$ of a tissue constraint point placed inside a tetrahedron is given by the barycentric coordinates and the displacement $\Delta \mathbf{q}_{t}$ of the 4 nodes $\mathbf{u}_{t}=\mathbf{J}_{t} \Delta \mathbf{q}_{t}$. The displacement of the needle point $\mathbf{u}_{n}$ is mapped using the interpolation of the needle deformation model $\mathbf{u}_{n}=\mathbf{J}_{n} \Delta \mathbf{q}_{n}$.

\section{$2.2 \quad$ Puncturing Soft Tissue}

Puncturing illustrates the interest of using complementarity theory to model the constraint: three successive steps of the interaction can be defined with a set of inequalities, as illustrated in Fig. 2. Here, $Q$ is the tip of the needle and $P$ is the contacting point (or the penetration point) on the tissue surface. $\mathbf{n}$ is the surface normal vector at point $P$.

The puncture constraint can be applied several times during the simulation if the needle passes through different tissue layers: different values for the threshold $f_{p}$ can be defined in order to simulate different tissue behaviors. If the tip of the needle hits a bone for example, $f_{p}$ is very high and never reached by $\lambda_{p}$ : the needle slips along the surface of the bone. The constraint is associated with an other constraint on each lateral direction: friction constraint when contact is established (step 2), and tip path constraint (see section 2.4) when puncture states are activated (step3).

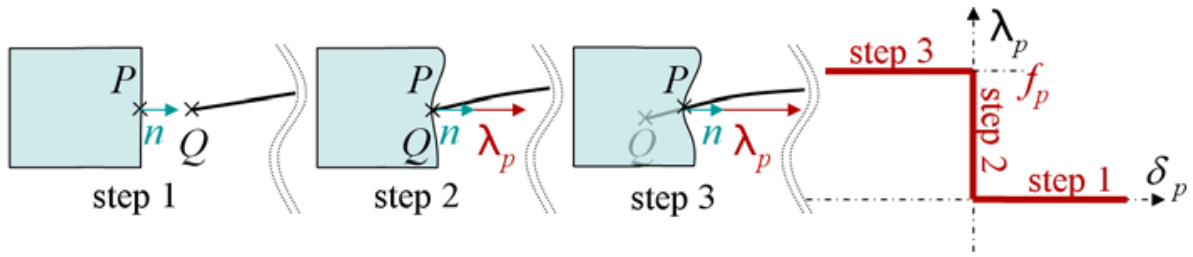

Fig. 2. Puncturing steps. During step 1, $Q$ is only approaching the tissue surface. The gap $\delta$ is positive $\left(\delta_{p} \geq 0\right)$ and the interaction force must be null $\left(\lambda_{p}=0\right)$. During step $\mathbf{2}, Q$ is touching without puncturing the tissue surface. The gap between $P$ and $Q$ is null $\left(\delta_{p}=0\right)$ and the interaction force is necessarily positive in the direction of the surface normal $\left(\lambda_{p} \geq 0\right)$. The value of this force is strictly less than a puncturing force threshold $\lambda_{p} \leq f_{p}$. During step 3, the needle tip enters in the tissue, the gap along the constraint direction is negative $\left(\delta_{p} \leq 0\right)$ and the constraint force is equal to the threshold $\left(\lambda_{p}=f_{p}\right)$.

\subsection{Cutting through Different Layers}

The cutting force $f_{c}$ is the force needed to traverse a tissue structure. As for $f_{p}$, it can be tuned to different values from a layer to another. This force disappears 
if the needle is re-inserted at the same location. The constraint used to simulate cutting is similar to the one used for puncturing, except that $\delta_{c}$ measures the relative displacement between the needle tip and the extremity of a curve created by a previous cutting path. $\lambda_{c}$ is still the force that solves the constraint.

\subsection{Tip Path and Needle Steering}

A direction is associated to the needle tip in order to constrain its lateral motion. To obtain needle steering due to bevel-tip needle, a specific orientation of the cutting direction in the tip frame is defined (Fig. 3(a)) and the displacement of the tip is tangential to this direction $\left(\delta_{t}=0\right)$. In that case, whether the needle is being pushed or pulled, the constraint is not aligned in the same direction (Fig. 3(b)). During needle insertion, the path of the tip inside the soft tissues is followed by the rest of the needle. This behavior is modeled using additional constraints: we impose a null relative displacement $\delta_{t}$ between the needle and the organ along the tangential directions of the needle curve (Fig. 3(c)). Here $\lambda_{t}$ will provide the force necessary to solve this equality constraint.
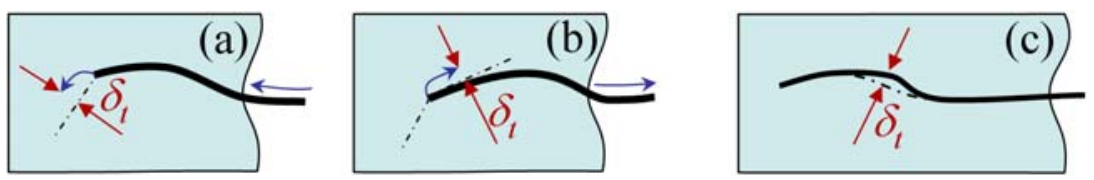

Fig. 3. Needle model is constrained on the path defined by the needle tip

\subsection{Friction}

Dry friction resists to the motion when the needle is inserted but also retracted. The complementarity constraint for the friction defines two states: adherence (stick) when there is no relative motion $\delta_{f}=0$ due to static friction and dynamic friction (slip) when the relative motion is not null $\delta_{f} \neq 0$ (Fig. 4).

A threshold $\mu . p$ is used to limit adherence: $\mu$ is the coefficient of friction and $p$ is the pressure exerted by the soft tissue on the needle. Currently, this pressure is estimated from the stiffness of the soft tissue and remains constant. To model it more accurately, we plan to use stress measures based on the soft tissue deformable model. The value of the friction resistance $r$, given by the graph (Fig. 4) is integrated along the inserted surface of the needle. Each constraint point owns a part of the needle curve and computes the friction force by using the length $l$ of this curve part $\left(\lambda_{f}=l \pi d . r\right.$ where $d$ is the diameter of the cross-section).
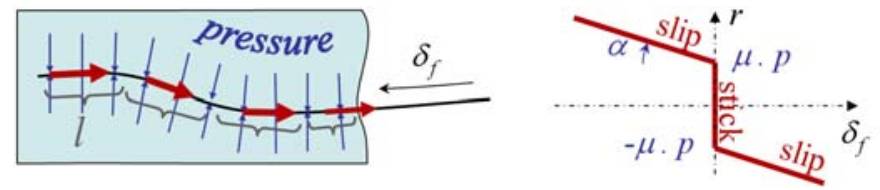

Fig. 4. Friction model: static and dynamic friction are included 


\section{Simulation of Tissue and Needle Interaction}

In this section, we describe how we combine, using Lagrangian mechanics, the previously defined insertion constraints with the deformation models of the needle and the soft tissues. Let's consider the dynamic deformation models of the needle $n$ and the soft tissue $t$. We use the synthetic formulation:

$$
\begin{array}{r}
\mathbf{M}_{n} \dot{\mathbf{v}}_{n}=\mathbf{p}_{n}-\mathbb{F}_{n}\left(\mathbf{q}_{n}, \mathbf{v}_{n}\right)+\mathbf{H}_{n}^{T} \lambda \\
\mathbf{M}_{t} \dot{\mathbf{v}}_{t}=\mathbf{p}_{t}-\mathbb{F}_{t}\left(\mathbf{q}_{t}, \mathbf{v}_{t}\right)+\mathbf{H}_{t}^{T} \lambda
\end{array}
$$

where $\mathbf{q} \in \mathbb{R}^{n}$ is the vector of generalized degrees of freedom, $\mathbf{M}$ is the mass matrix, $\mathbf{v} \in \mathbb{R}^{n}$ is the vector of velocity. $\mathbb{F}$ represents internal visco-elastic forces, and $\mathbf{p}$ gathers external forces. $\lambda$ is the vector of the constraint forces that is multiplied by matrices $\mathbf{H}_{n}^{T}$ and $\mathbf{H}_{t}^{T}$ presented on section 2.1

To allow a stable interaction between models, implicit integration is used with backward Euler scheme. The simulation is realized in three steps: during the first step, constraint forces are ignored to obtain what we call a free motion of each model. During the second step, constraint forces $\lambda$ are computed as described in the following. Then when $\lambda$ is known, a correction of the motion of each model is performed in order to respect equations (3) and (4).

The computation of the contact forces, during step 2, relies on solving the constraint laws presented on section 2 with the following equation:

$$
\boldsymbol{\delta}=[\underbrace{\mathbf{H}_{n}\left(\frac{\mathbf{M}_{n}}{h^{2}}+\frac{d \mathbb{F}_{n}}{h d \mathbf{v}_{n}}+\frac{d \mathbb{F}_{n}}{d \mathbf{q}_{n}}\right)^{-1} \mathbf{H}_{n}^{T}}_{\mathbf{W}_{n}}+\underbrace{\mathbf{H}_{t}\left(\frac{\mathbf{M}_{t}}{h^{2}}+\frac{d \mathbb{F}_{t}}{h d \mathbf{v}_{t}}+\frac{d \mathbb{F}_{t}}{d \mathbf{q}_{t}}\right)^{-1} \mathbf{H}_{t}^{T}}_{\mathbf{W}_{t}}] \lambda+\boldsymbol{\delta}^{\text {free }}
$$

where $h$ is a time step and $\left(\frac{\mathbf{M}}{h^{2}}+\frac{d \mathbb{F}}{h d \mathbf{v}}+\frac{d \mathbb{F}}{d \mathbf{q}}\right)$ is the dynamic tangent matrix. We note that depending on the deformation model, the computation of matrices $\mathbf{W}$ could be time consuming (see discussion on section 4). To find the constraint force value, we propose to use a Gauss-Seidel like algorithm. Considering a constraint $\alpha$, among $m$ instantaneous constraints, one can rewrite equation (5):

$$
\delta_{\alpha}-\left[\mathbf{W}_{\alpha, \alpha}\right] \lambda_{\alpha}=\underbrace{\sum_{\beta=1}^{\alpha-1}\left[\mathbf{W}_{\alpha, \beta}\right] \lambda_{\beta}+\sum_{\beta=\alpha+1}^{m}\left[\mathbf{W}_{\alpha, \beta}\right] \lambda_{\beta}+\delta_{\alpha}^{\text {free }}}_{\delta_{\alpha}^{-}}
$$

where $\left[\mathbf{W}_{\alpha, \beta}\right]$ is the value of the matrix $\mathbf{W}=\mathbf{W}_{n}+\mathbf{W}_{t}$ at line $\alpha$ and column $\beta$. It models the coupling between constraint points $\alpha$ and $\beta$. At each iteration of the Gauss-Seidel solver, each constraint $\alpha$ is visited and a new estimate of $\lambda_{\alpha}$ is performed while "freezing" the contributions of $\lambda_{\beta}$ with $\alpha \neq \beta$. The new estimate of $\lambda_{\alpha}$ is found at the intersection of the characteristic graph of each constraint law described in section 2 with the line of equation (6). Note that for all mechanical models, the local compliance of a point is always positive : 

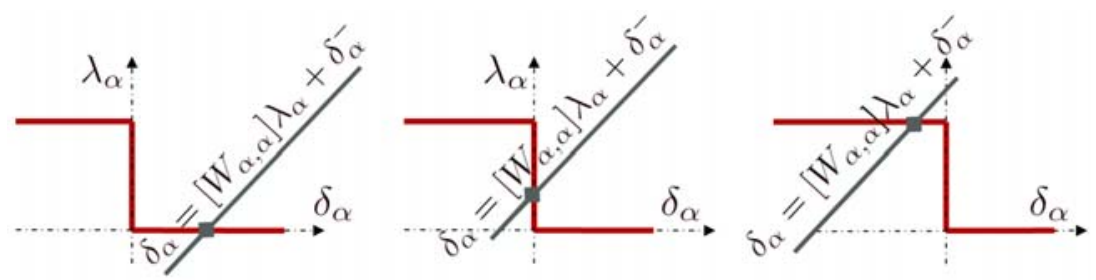

Fig. 5. Example of graph intersection to find $\lambda_{\alpha}$ on a given constraint graph

$\left[W_{\alpha, \alpha}\right]>0$, so we obtain a unique solution for $\lambda_{\alpha}$ (see Fig [5). We stop the algorithm when the error on the values of vector $\boldsymbol{\delta}$ reach a given threshold.

In our approach, the sampling of the constraints is important for both accuracy and convergence of the constraint solver. Namely, if there are more constraints than degrees of freedom involved, the problem is over-constrained. However, if the space between the constraints points is too large, the accuracy decreases. In practice, we use a regular sampling of the constraint points along the needle curve that corresponds to the discretization of the needle deformation model. During the insertion and the withdrawal of the needle, constraint points are dynamically inserted and removed to obtain a sufficient number of constraints along the needle curve.

\section{Results}

\subsection{Deformable Models}

In this work, the needle model that relies on a series of beam elements can handle deformations with large displacements. The parameters of the model are mainly: $E$, the Young Modulus, $\nu$ the poisson ratio, and $A$ the area of the cross section (hollow needles can be simulated). The computation is optimized using a band tri-diagonal solver: $\mathbf{W}_{n}$ value is obtained at high refresh rate (few milliseconds) with a precise discretization of the needle (50 beams).

The soft tissues of the human anatomy often have a visco-elastic anisotropic behavior which leads to complex FEM models if high precision is needed. However, to assess our constraint-based model of the interaction between the needle and the soft tissues during insertion, we use basic shapes and simple tissue model. The simulated soft tissue can only undergo large displacements with small deformations. We use Hooke's constitutive law: deformations are considered to be isotropic and elastic and the Poisson ratio is tuned to quasi incompressibility $(\nu=0.49)$. A viscous behavior is obtained using Rayleigh model. It provides an attenuation that is proportionally related to both elastic and inertial forces. Using this model, we can obtain a very fast estimation of the compliance matrix $\mathbf{W}_{t}[10$, that leads to the ability of obtaining real-time simulation of the needle insertion. Once again, this tissue model is only used in a preliminary approach, to validate the constraints used for needle insertion. More complex model could be used. 


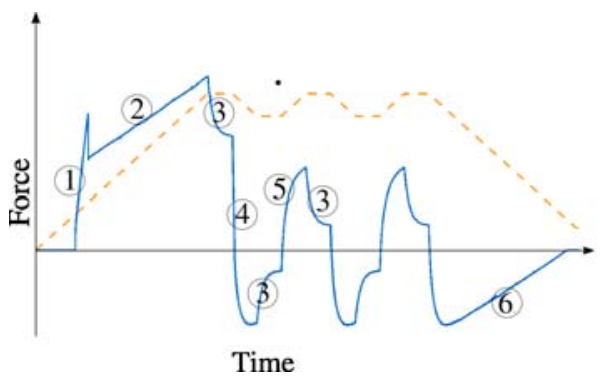

Fig. 6. Insertions and pullbacks. Stippled line represents the motion imposed to the needle. At step (1), the needle punctures the tissue surface. During step (2), friction is increasing with the penetration distance and (3) is the relaxation. After being partially retracted (4), the needle is inserted again along the same path (5); therefore no cutting force is applied. During the last and complete pullback (6), the friction force decreases.

\subsection{Experiments}

Our first experiment consists in inserting and pulling back multiple times the needle in a 3D soft tissue. This experiment is similar to the measurements proposed in [8], and the results obtained with our simulation (presented in Fig. 6) match the previous work.

Then we propose a second experiment based on the 3D simulation of an obstacle avoidance using needle steering. Indeed, surgeons sometimes use thin and flexible needles with beveled tip to reach the target with a curved path. However, flexible needle insertion and navigation deep into the tissue complicate the procedure [1]. The experiment is close to the one presented in [12], except that their tissue phantom was considered as rigid and here the tissue is deformable. The Fig. 7 shows a real-time simulation of needle steering in a deformable object (we obtain an average of 28 frames per second).

In-vivo tissues are inherently non-homogeneous and thin medical tools can be deflected by harder regions inside overall soft tissues. We simulate this phenomenon using a 3D volume mesh composed of two regions with different stiffnesses. During its insertion, a needle collides with the surface of the stiff region: as the force at the tip is lower than the puncture threshold, the needle slides along the surface of this object. Using the same parameters, but with an

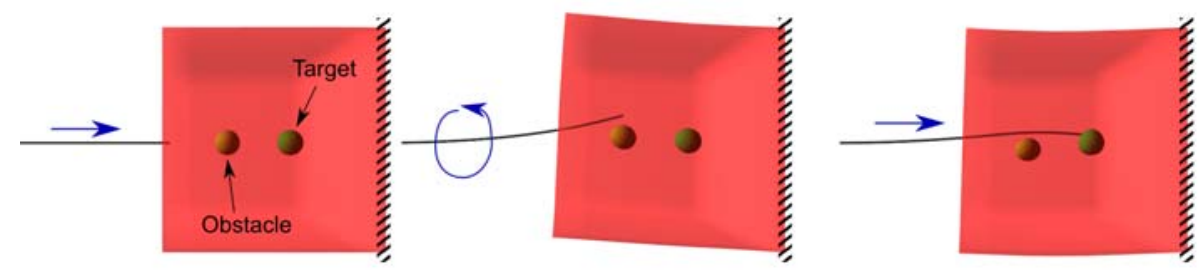

Fig. 7. The needle is first inserted one half of the distance into the phantom, then spun 180 degrees, and finally inserted the remaining distance 


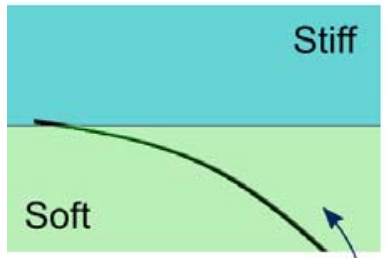

Soft needle

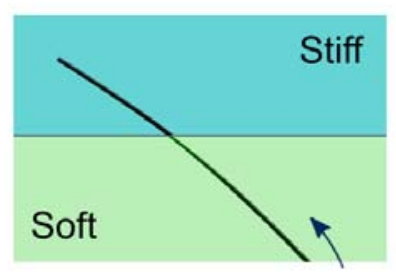

Stiff needle

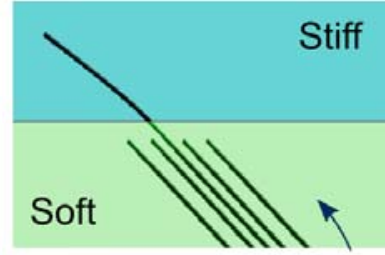

Multiple soft needles

Fig. 8. Needle deviation when puncturing nonhomogeneous layers. (Left) the needle cannot penetrate the harder inclusion. (Center) First solution: by increasing the stiffness of the needle, it can now puncture the second region. (Right) Second solution: additional needles are inserted into the soft tissue, the flexible needle can penetrate.

increase of its stiffness, the needle punctures the stiff region, as shown in Fig. 8 , The soft region can also be rigidified by the insertion of other needles. It allows for a precise insertion of the flexible needle without increasing its stiffness. This technique is commonly used for brachytherapy.

\section{Conclusion and Future Work}

In this paper, we demonstrate the interest of using complementarity constraints for the simulation of the insertion of flexible needles into soft tissue. The presented model can be parameterized using previous work experiments, and also allows for the simulation of more complex scenarios.

We plan to complete our simulations using more realistic constitutive laws for the deformation of the anatomy. In the near future, we will perform some validations on experiments to assess the precision on scenarios that are closer to clinical procedures. We aim at using the simulation as a planning tool for some therapeutic protocols that rely on the insertion of slender medical instruments.

Acknowledgments. This work is supported by ANR project 06-MDCA-015 VORTISS.

\section{References}

1. DiMaio, S., Salcudean, S.: Needle steering and motion planning in soft tissues. IEEE Transactions on Biomedical Engineering 19(6), 965-974 (2005)

2. Alterovitz, R., Goldberg, K., Okamura, A.: Planning for steerable bevel-tip needle insertion through $2 \mathrm{~d}$ soft tissue with obstacles. In: Proceedings of ICRA 2005, pp. 1652-1657 (2005)

3. Abolhassani, N., Patel, R., Moallem, M.: Needle insertion into soft tissue: A survey. Medical Engineering and Physics 29, 413-431 (2007)

4. Marchal, M., Promayon, E., Troccaz, J.: Comparisons of needle insertion in brachytherapy protocols using a soft tissue model. In: Proceedings of the Third International Conference Surgetica 2007, pp. 153-160 (2007) 
5. Okamura, A., Simone, C., O'Leary, M.: Force modeling for needle insertion into soft tissue. IEEE Transactions on Biomedical Engineering 51(10), 1707-1716 (2004)

6. Crouch, J.R., Schneider, C.M., Wainer, J., Okamura, A.M.: A velocity-dependent model for needle insertion in soft tissue. In: Duncan, J.S., Gerig, G. (eds.) MICCAI 2005. LNCS, vol. 3750, pp. 624-632. Springer, Heidelberg (2005)

7. Hing, J., Brooks, A., Desai, J.: A biplanar fluoroscopic approach for the measurement, modeling and simulation of needle and soft-tissue interaction. Medical Image Analysis 11, 62-78 (2007)

8. Dehghan, E., Wen, X., Zahiri-Azar, R., Marchal, M., Salcudean, S.: Needle-tissue interaction modeling using ultrasound-based motion estimation: Phantom study. Computer Aided Surgery 13(5), 265-280 (2008)

9. Murty, K.G.: Linear Complementarity, Linear and Nonlinear Programming, Internet edition. (1988)

10. Saupin, G., Duriez, C., Cotin, S., Grisoni, L.: Efficient contact modeling using compliance warping. In: Proceedings of CGI 2008 (2008)

11. Glozman, D., Shoham, M.: Flexible needle steering and optimal trajectory planning for percutaneous therapies. In: Barillot, C., Haynor, D.R., Hellier, P. (eds.) MICCAI 2004. LNCS, vol. 3217, pp. 137-144. Springer, Heidelberg (2004)

12. Webster, R., Kim, J., Cowan, N., Chirikjian, G., Okamura, A.: Nonholonomic modeling of needle steering. International Journal of Robotics Research 5(6), 509-525 (2006) 